\title{
水滑石多尺度结构精准调控及其光驱动催化应用研究
}

\author{
许艳旗 ${ }^{1}$, 谭玲 ${ }^{1}$, 王泽林 ${ }^{1}$, 郝晓杰 ${ }^{1}$, 王纪康 ${ }^{1}$, 赵宇飞 ${ }^{1 *}$, 宋宇飞 ${ }^{1,2^{*}}$
}

1. 北京化工大学, 化工资源有效利用国家重点实验室, 北京 100029 ;

2. 北京化工大学北京软物质科学与工程高精尖创新中心, 北京 100029

*联系人, E-mail: zhaoyufei@mail.buct.edu.cn; songyf@mail.buct.edu.cn

2018-08-15 收稿, 2018-09-02 修回, 2018-09-05 接受, 2018-09-26 网络版发表 国家重点研发计划(2017YFB0307303)、国家重点基础研究发展计划(2014CB932104)、国家自然科学基金(U1707603，21625101，21521005, U1507102)、北京市自然科学基金(2182047)和中央高校基金(ZY1709)资助

摘要水滑石(LDHs)具有独特的层状结构、可调的组成、可控的粒径大小和形貌、简单的合成方法、易于放大 生产制备等特点，使其具有丰富的可调控性; 进一步经过拓扑转变，可制备得到复合金属氧化物、负载型金属催 化剂，为设计具有能带结构适宜、表界面结构丰富的绿色、高效纳米催化剂提供了广阔的平台。针对温和条件下 $\mathrm{H}-\mathrm{O}, \mathrm{C}=\mathrm{O}, \mathrm{N} \equiv \mathrm{N}, \mathrm{C}-\mathrm{C}$ 键光驱动催化活化, 本文围绕“水滑石多尺度结构精准调控及催化过程强化”的主题, 通过 对水滑石微观精细结构、介观异质界面结构、高分散催化剂分散特性等方面进行精准调控，实现了水滑石基催化 剂在光驱动分解 $\mathrm{H}_{2} \mathrm{O}$, 还原 $\mathrm{CO}_{2}, \mathrm{CO}$ 加氢制高碳烃、合成氨以及降解环境污染物等反应的强化; 揭示了基于能带、 缺陷、界面可控的水滑石基材料制备-结构-光驱动催化性能之间的关系, 从而为实现太阳能高效转化提供思路.

关键词水滑石, 多尺度结构调控, 缺陷, 界面, 光催化, 光热催化

多相催化在国民经济中占有重要地位，85\%的化 学工业产品都有催化剂参与. 催化剂的性能与其制 备过程、结构密不可分, 而目前催化剂制备方法和技 术较为成熟, 主要包括沉淀法、浸渍法、溶液蒸干法、 热熔融法、离子交换法等, 但这些方法和工艺也有局 限性, 如活性组分容易团聚烧结、活性组分分散不均 匀、活性组分容易被包埋等, 进而限制了催化效率的 进一步提升. 此外, 开发绿色、高效、可持续、可替 代化石能源的催化新工艺路线, 已成为化学工程等 相关学科研究的焦点. 为了应对上述问题, 发展新型 催化剂制备方法, 在分子层次揭示、优化活性位结构, 介观层次研究催化剂界面结构, 实现催化剂活性组 分高分散, 以此来提高催化剂的活性和稳定性, 是目 前提高催化效率的必然途径 ${ }^{[1,2]}$.

水滑石 $(\mathrm{LDHs})$ 是一类独特的二维层状双氢氧化
物, 其分子式为 $\left[\mathrm{M}_{1-x}^{2+} \mathrm{M}_{x}^{3+}(\mathrm{OH})_{2}\right]^{x+}\left[\mathrm{A}_{x / n}\right]^{n-} \cdot m \mathrm{H}_{2} \mathrm{O}$, LDHs主体层板结构类似于水镁石 $\mathrm{Mg}(\mathrm{OH})_{2}$, 由八面 体金属氧化物 $\mathrm{MO}_{6}$ 共边连接, 主体层板金属元素高 度有序排列 ${ }^{[3]}$. 这些层板上的部分二价金属阳离子 $\left(\mathrm{M}^{2+}\right)$ 被三价金属阳离子 $\left(\mathrm{M}^{3+}\right)$ 取代, 使得层板带正电, 其中 $\mathrm{M}^{2+}, \mathrm{M}^{3+}$ 可以为 $\mathrm{Mg}, \mathrm{Al}, \mathrm{Ti}, \mathrm{V}, \mathrm{Cr}, \mathrm{Mn}, \mathrm{Fe}, \mathrm{Co}, \mathrm{Ni}$, $\mathrm{Cu}, \mathrm{Zn}, \mathrm{Ga}$, In等元素中的一种或几种; 层板所带的 正电荷由层间阴离子 $\left(\mathrm{A}^{n-}\right)$ 来平衡. $\mathrm{M}^{2+}, \mathrm{M}^{3+}$ 在平面上 高度原子级交替排列构成了水滑石的平面结构 $(a, b$ 轴方向), 带正电荷的层板和带负电的客体阴离子层 相互交替 ( $c$ 轴方向), 构成了 LDHs 的立体结构 ${ }^{[3,4]}$. LDHs独特的结构特点, 例如主体层板元素相互高分 散、晶粒尺寸和层板厚度可控、自身在结构转变过程 中具有拓扑转变的特性等, 为LDHs带来了丰富的可 调变性 ${ }^{[5,6]}$. 其中, LDHs的主体层板元素以原子级交 
替有序排列的特性, 能够将具有催化活性的过渡金 属元素(如Fe, Co, Ni, Ti等)高度分散于LDHs的层板 中, 可直接用作光催化剂, 提高活性组分的原子利用 率. 另外, LDHs的拓扑转变特性可以使LDHs材料在 空气或还原气氛下高温焙烧转为特定形貌的异质金 属氧化物或负载型高分散金属催化剂 ${ }^{[7,8]}$; 而基于 LDHs晶粒尺寸和层板厚度可调的特点, 可以在微纳 米尺度上调控LDHs以及LDHs拓扑转变衍生物的粒 径、厚度、形貌等结构成为可能, 可以充分暴露活性 位点, 进而提升催化性能 ${ }^{[9,10]}$.

太阳能光催化技术具有常温常压深度反应、可直 接利用太阳能作为能量来源来驱动反应等独特优势, 已经成为一种理想的洁净能源生产途径和环境治理 新技术而受到学术界和产业界广泛瞩目. 基于LDHs 材料丰富的可调控特性, 能够实现对其组成、尺寸、 缺陷、界面以及能带等结构方面的精准调控. 本文系 统综述了水滑石微观精细结构、介观异质界面结构、 高分散催化剂分散特性等方面的最新进展(图1), 及 其在光驱动分解水、催化还原 $\mathrm{CO}_{2}, \mathrm{CO}$ 加氢制高碳 烃、合成氨以及处理环境污染物等方面的应用 ${ }^{[5,6,11]}$, 揭示水滑石基纳米材料制备-结构-光驱动催化转化 性能之间的关系, 为实现高效太阳能转化提供思路.

\section{1 水滑石结构调控及认知}

\section{1 厚度和尺寸调控的最新进展}

随着纳米技术的发展, 对于尺寸和形貌的调控 越来越受到人们的重视和研究 ${ }^{[12]}$. 通过新的合成方 法和策略可以实现LDHs的可控合成. 对晶粒尺寸和 层板厚度的调控, 可以有效调变水滑石的物理化学 性质, 使得水滑石获得更加奇特的性能. 相比传统块 体LDHs, 尺寸和厚度精准调控的LDHs展示了极其
广阔的应用前景 ${ }^{[10,13]}$. 基于LDHs 主体层板的强极性 特点, 采用水溶剂法合成的LDHs粒径大都在500 nm 以上, 且层板堆叠严重. 2012年, Zhao等人 ${ }^{[14]}$ 通过共 沉淀法合成了一系列 MTi-LDHs(M=Ni, Zn, MgAl). 合成的水滑石其晶粒尺寸为 $200 \sim 500 \mathrm{~nm}$, 层板厚度 为20 40 nm. 为了避免层板堆叠带来活性位点少的 问题, 2013年采用反向微乳法成功合成了晶粒尺寸分 布较窄(40 80 nm), 层板厚度 9 $\mathrm{nm}$ 的ZnTi-LDH纳米 片 $^{[15]}$. 2014年, 通过精细调控微乳合成方法, 成功合 成了晶粒尺寸 30 60 $\mathrm{nm}$, 层板厚度为 $2 \mathrm{~nm}(1 \sim 2$ 层) 的 NiTi-LDH超薄纳米片 ${ }^{[16]}$. 进一步地, 通过对微乳 液尺寸进行调控, 张铁锐课题组 ${ }^{[17]}$ 成功合成了晶粒 尺寸分别为 $2 \mu \mathrm{m}$ 和 $20 \mathrm{~nm}$, 层板厚度分别为 $\sim \mu \mathrm{m}$ 和 0.9 nm的NiTi-LDH(图2(a) (d)). 近期, 张铁锐课 题组 ${ }^{[18]}$ 通过对单层 NiFe-LDH进行超声粉碎, 第 1 次 成功合成了平均晶粒尺寸约为 $2.3 \mathrm{~nm}$, 层板厚度为 $0.6 \mathrm{~nm}$ 的超小单层 $\mathrm{NiFe}-\mathrm{LDH}$ (图2(e), (f)). 该 $\mathrm{LDH}$ 是 目前文献报道的最小的无载体 LDH. 迄今, 通过对 LDHs形成环境的调控, 可以实现对其粒径 $2.3 \mathrm{~nm} 3$ $\mu \mathrm{m}$, 厚度从单层到数百层厚度的调控. 通过对 LDHs 纳米结构的精准调控, 为下一步拓扑结构转变提供 了丰富的结构支撑.

\section{2 纳米尺寸效应}

随着 LDHs纳米材料粒径以及厚度的减少, 不可 避免的产生一些扭曲结构以及缺陷结构. 而这些结 构往往和催化活性和选择性有着紧密联系. 通过多 种谱学手段去认识和理解这些结构, 有助于理解催 化剂结构和催化反应的构效关系. X射线精细结构吸 收谱(XAFS)技术对中心吸收原子的化学环境和局域 结构敏感, 是一种研究原子的近邻结构和电子结构的 重要方法 ${ }^{[19]}$. 通过XAFS表征, 可以对水滑石的配位

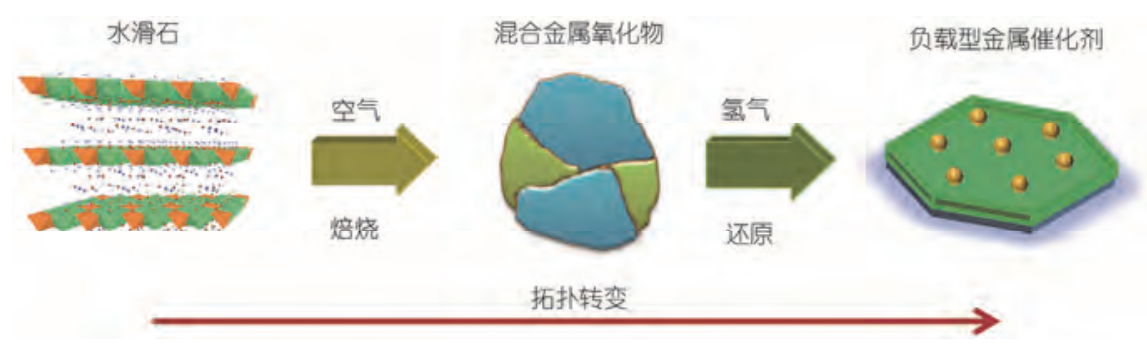

图 1 (网络版彩色)水滑石拓扑结构转变制复合氧化物以及高分散金属催化剂的模型结构图

Figure 1 (Color online) Model structure diagram of topological transformation of LDHs for the preparation of mixed metal oxides and highly dispersed metal catalysts 

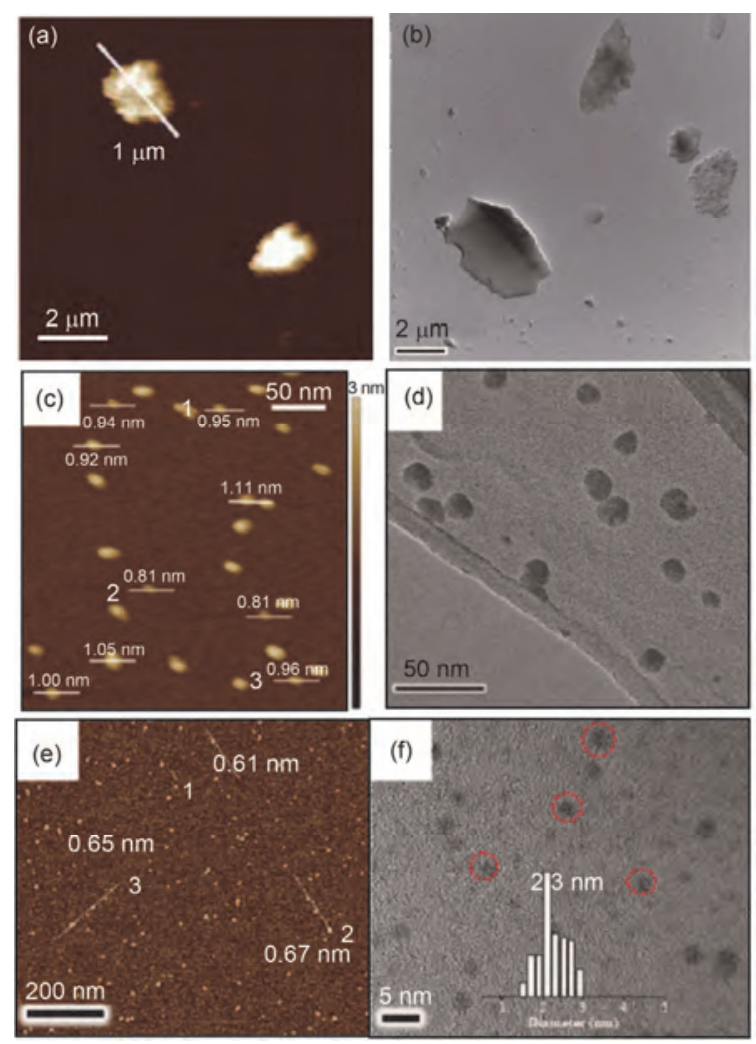

图 2 (网络版彩色)大块和单层NiTi-LDH的AFM图(a, c) 和TEM图(b, d) ${ }^{[17]}$; 超小单层NiFe-LDH的AFM图(e)和TEM图(f)以及粒径分布图 ${ }^{[18]}$

Figure 2 (Color online) AFM (a, c), TEM (b, d) images of bulk and monolayer NiTi-LDH nanosheets ${ }^{[17]}$; AFM (e) and TEM (f) images and corresponding particle size distribution of ultrafine monolayer NiFeLDH nanosheets ${ }^{[18]}$

结构、缺陷结构、层板阳离子的电子结构等进行研究. 2014年, Zhao等人 ${ }^{[16]}$ 利用反向微乳液的方法成功合成 了超薄NiTi-LDH(NiTi-LDH-1, 粒径大小 30 nm), 并 与大块NiTi-LDH(NiTi-LDH-4, 粒径大小 2 $\mu \mathrm{m}$ )作为 对照, 对其Ti边进行了XAFS测试. 通过XAFS表征, 发现NiTi-LDH-1的白线峰位置相比于NiTi-LDH-4向 低角度移动了 $1.1 \mathrm{eV}$, 说明 NiTi-LDH-1 中存在 $\mathrm{Ti}^{3+}$, 进一步通过对 R空间拟合发现相比于 NiTi-LDH-4, NiTi-LDH-1具有更长的 Ti-O键, 更低的配位数以及 更大的Debye-Waller因子, 这些都表明NiTi-LDH-1中 存在结构扭曲以及配位不饱和.

2017年, 张铁锐课题组 ${ }^{[20]}$ 利用XAFS技术对大块 $\mathrm{CuCr}-\mathrm{LDH}$ 和超薄CuCr-LDH(大小 20 nm, 厚度 2.5 $\mathrm{nm}$ ) 进行了更深人的研究, 发现超薄 $\mathrm{CuCr}-\mathrm{LDH}$ 在 $5993 \mathrm{eV}$ 处存在明显的峰, 说明存在配位位点的无序 和形变(图 S1(a), (b)), 并且超薄 $\mathrm{CuCr}-\mathrm{LDH}$ 中 Cr的配 位环境也和大块 $\mathrm{CuCr}-\mathrm{LDH}$ 有所不同, 超薄 $\mathrm{CuCr}-$
$\mathrm{LDH}$ 中具有更短的 $\mathrm{Cr}-\mathrm{O}$ 键长 $(1.989 \AA)$, 大块 $\mathrm{CuCr}-$ $\mathrm{LDH}$ 为 $1.990 \AA$ ), 更低的配位数 $(5.5$, 大块 $\mathrm{CuCr}-\mathrm{LDH}$ 为 6.0), 这些都表明了超薄 CuCr-LDH中存在丰富的 空位结构 (图 S1(d)). 同时, 相比大块 CuCr-LDH中 $\mathrm{Cr}-\mathrm{Cu}$ 键键长 (3.056 ̊), 超薄 $\mathrm{CuCr}-\mathrm{LDH}$ 中 $\mathrm{Cr}-\mathrm{Cu}$ 键 键长(3.060 ̊) 变大, 配位数降低, 表明了超薄水滑石 在 $a, b$ 平面上发生了结构扭曲变形, 并且通过XRD测 试也发现CuCr-LDH, ZnAl-LDH纳米片相比其元素组 成比例一致的 LDH块体而言, 除了 (001) 峰变弱外, (110) 峰向高角度移动证明了 LDH纳米片晶胞参数 $a\left(a=2 \mathrm{~d}_{(110)}\right)$ 分别减少 $0.2 \%, 0.5 \%$ (图 S1(e)). 上述结构 显示随着LDHs形貌纳米化, 其存在的表面缺陷带来 了结构扭曲, 并伴随有晶胞收缩, 存在不同程度的应 变效应.

此外, 缺陷和结构扭曲的产生对金属价态也有 影响. 通过研究NiTi-LDH发现随着粒径和厚度的减 少, XPS和ESR结果都证明除了自身存在大量的 $\mathrm{Ni}^{2+}$, $\mathrm{Ti}^{4+}$ 外, 因为氧缺陷的存在, 形成了大量的 $\mathrm{Ni}^{3+}$ 和 $\mathrm{Ti}^{3+}$. 这种变价金属掺杂的 $\mathrm{LDH}$, 对其导电性等方面 起到了重要作用. 此外, 围绕ZnAl-LDH纳米片, 张 铁锐课题组 ${ }^{[21]}$ 合成了超薄ZnAl-LDH, 和大块 ZnAl$\mathrm{LDH}$ 相比, 电子顺磁共振谱(EPR) 在 $g=1.998$ 处出现 了明显的信号 (图 S2(a), (b)), 由此证明了超薄 $\mathrm{ZnAl}-\mathrm{LDH}$ 中存在 $\mathrm{Zn}^{+}-\mathrm{V}_{\mathrm{O}}$ 和配位不饱和 $\mathrm{Zn}$ 的形成. 围 绕LDH拓扑结构转变, 2016年张铁锐课题组 ${ }^{[22]}$ 利用 反相微乳法合成了单层的 NiTi-LDH, 并以此为前体 焙烧得到Mono-NiTi-MMO. 由 Ti 2 及及Ni $2 p$ 的XPS测 试结果可知(图S2(c), (d)), 相比于大块的Bulk-NiTiMMO, Mono-NiTi-MMO有更多的 $\mathrm{Ti}^{3+}$ 及 $\mathrm{Ni}^{3+}$, 这可能 是由于Mono-NiTi-MMO的焙烧前体为单层, 具有更 多的缺陷结构, 煅烧过程中依旧保持了上述结构的 特点. 综上, 围绕LDH纳米结构, 随着减小厚度和尺 寸, 其结构不可避免存在扭曲和氧缺陷, 进而导致水 滑石中金属价态、结构、晶胞参数等有所改变. 这对 后续研究催化剂和性能之间的构效关系奠定了坚实 的基础.

\section{2 水滑石拓扑结构转变制备高活性晶面取 向的异质界面结构}

拓扑和拓扑转变研究的是在连续变化空间中保 持不变的空间构型可定向性的内在性质. 晶体学中 的拓扑转变反应是指同一反应中, 反应物晶体和产 
物晶体存在至少一种晶体结构上的等价关系. 该等 价关系可以晶体尺寸相等, 也可以是晶型统一. 这些 特性来源晶体内部原子排布方式拓扑不变性, 又称 为 “局域规整反应”. 拓扑转变以及拓扑材料方面的 理论发现为后来拓扑材料的出现奠定了基础, 揭开 了人类研究材料转变的新篇章. 为此, 2016年度诺贝 尔物理学奖颁给3位英美科学家David J. Thouless, F. Duncan M. Haldane和J. Michael Kosterlitz, 以奖励他 们在拓扑相变以及拓扑材料方面的理论发现. 在催 化剂制备方面, 拓扑转变对于理解催化剂制备过程 以及结构转变具有重要指导作用. LDHs作为一类主 体层板金属元素高度有序排列和组成可调的二维层 状材料, 可利用其拓扑转变性质制备特定形貌的混 合金属氧化物. 混合金属氧化物间形成的界面被证 明是促进电荷分离和电子转移的活性位点, 同时暴 露的高活性晶面可提高催化活性. 因此通过LDHs拓 扑转变制备具有丰富异质结界面, 高活性晶面暴露 的混合金属氧化物是提高催化活性的一种有效手段. LDHs 的拓扑转变现象最初是 1990年由 Markov和 Petrov $^{[7]}$ 报道, 通过焙烧 $\mathrm{CuCo}-\mathrm{LDH}$ 制备了 $\mathrm{CuCo}$ 尖晶 石, 发现其(111)面和LDHs的(001)面存在着类似的原 子分布, 因而认为LDHs至尖晶石的热分解过程是一 种拓扑转变机理. Xiang等人 ${ }^{[8]}$ 和Zhao等人 ${ }^{[23,24]}$ 对不 同主体层板元素组成的LDHs的拓扑结构转变也进行 了大量的研究, 发现利用拓扑转变, 将LDHs前体进 行焙烧可以制备活性晶面暴露的高分散负载型纳米 催化剂. 所获得的催化剂不仅能够保持LDHs前体的 形貌, 而且LDHs 主体层板的针定效应能够使拓扑转 变得到的催化剂保持较高的分散度和热稳定性. $\mathrm{He}$ 等人 ${ }^{[25]}$ 发现炦烧超薄ZnAl-LDH, 形成了(001)面暴露 的 $\mathrm{ZnO}$ 负载 $\mathrm{Al}_{2} \mathrm{O}_{3}$ 结构, 该活性晶面在光催化降解方 面具有促进作用. 张铁锐课题组 ${ }^{[22]}$ 和Zhao 等人 ${ }^{[26]}$ 通 过微乳液法合成了单层NiTi-LDH, 经过高温煅烧拓 扑转变合成了超薄超小 $\mathrm{NiO} / \mathrm{TiO}_{2}$ 异质纳米结构 (图 $3(\mathrm{a})$, (b)). 在 $\mathrm{NiO} / \mathrm{TiO}_{2}$ 异质结构中, 超薄的 $\mathrm{NiO}$ 纳米 片 ( 4 nm) 优先暴露了 (110)活性面, 而 $\mathrm{TiO}_{2}$ 优先暴露 (001) 晶面, 并伴随着大量的镍空位和丰富的异质结 界面, 进一步促进了电催化分解水产氧反应的进行.

2018年, 本课题组 ${ }^{[26]}$ 利用了水滑石的拓扑转变 性质, 进一步拓展了 $\mathrm{LDH}$ 的种类和性能. 通过拓扑 转变, 对含 $\mathrm{Co}$ 的LDHs进行焙烧可得到高活性晶面暴 露的 $\mathrm{Co}_{3} \mathrm{O}_{4}$ (图3(c)), 将该 $\mathrm{Co}_{3} \mathrm{O}_{4}$ 纳米片用于醇和胺的
无受体脱氢耦合反应中, 结果显示该催化剂在温和 条件下实现对上述反应 99\%的转化率和选择性. 在 此基础上, 以 $\mathrm{ZnCo}-\mathrm{LDH}$ 为前体, 对拓扑转变过程中 混合金属氧化物和异质结界面的形成及转变进行了 深人细致的研究(图4). $200^{\circ} \mathrm{C}$ 时 $\mathrm{ZnCo}-\mathrm{LDH}$ 层板结构 坄塌, 出现(112)晶面暴露的尖晶石结构和(001)晶面 暴露的 $\mathrm{ZnO}$ 结构, 并且晶粒之间存在着大量的异质 结界面. 随着焙烧温度从 200 到 $800^{\circ} \mathrm{C}$ 的升高, 晶粒之 间的异质结界面越来越清晰, 但异质结界面的比例 随着晶粒尺寸的增大而减少(图4). 通过调变拓扑温 度实现了异质结界面的调控, 改变了 $\mathrm{ZnO} / \mathrm{Co}_{3} \mathrm{O}_{4}$ 异质 结构的电子环境, 降低能带间隙, 进而促进电子传 递, 其电解水析氧性能获得了极大提高 ${ }^{[27]}$.

\section{3 水滑石拓扑转变制高分散金属催化剂}

\section{1 高分散 Ni, Co, Fe基异质结构催化剂的制备}

由于催化剂表面的活性组分高度分散, 其金属 的利用率非常高, 高催化活性、高分散金属催化剂在 资源利用上有着普通催化剂不具备的优势. 但是高 分散金属催化剂高温不稳定, 原子发生迁移(或团簇 迁移)从而团聚形成大颗粒，破坏原有的高分散状态， 影响催化剂的稳定性. LDHs具有晶格限域效应, 主 体层板上的二价和三价金属能够高度有序均匀排布, 可以用作高分散催化剂前体 ${ }^{[28]}$. LDHs 在高温脱水过 程中的独特拓扑转变效应, 有利于金属还原过程中 实现金属纳米颗粒高度分散. 围绕廉价 $\mathrm{Ni}, \mathrm{Co}, \mathrm{Fe}$ 基 高分散金属催化剂, 张铁锐课题组、马丁课题组和温 晓东课题组 ${ }^{[29]}$ 合作, 通过简单的煅烧-氢气还原方法, 将 $\mathrm{NiAl}-\mathrm{LDH}$ 载体在 $525^{\circ} \mathrm{C}$ 可控还原为 $\mathrm{NiO}_{x} / \mathrm{Ni}$ 纳米结 构, 提高温度, 大量的金属单质 $\mathrm{Ni}$ 颗粒被还原出来 (图5), 通过调控还原温度, 成功实现了 $\mathrm{NiO}_{x}$ 纳米层 部分针定 $\mathrm{Ni}$ 纳米颗粒的调控. 多种谱学手段揭示了 $\mathrm{NiO}_{x} / \mathrm{Ni}$ 纳米结构的生成过程, $\mathrm{NiO}_{x}$ 纳米片和 $\mathrm{Ni}$ 纳米 颗粒之间丰富的界面, 改变了 $\mathrm{NiO}_{x} / \mathrm{Ni}$ 纳米结构的电 子结构(图5和 S3). 该独特的结构实现了低温常压可 见光驱动 $\mathrm{CO}$ 加氢制备高级烃类, $\mathrm{C}_{2+}$ 选择性高达 $60 \%$, 且催化稳定性优越 ${ }^{[29]}$. 此外, 通过调控其他加氢催 化剂(如 $\mathrm{Co}, \mathrm{Fe}$ 基界面)利用 $\mathrm{LDH}$ 前体高度分散的特 性，在300 600 ${ }^{\circ} \mathrm{C}$ 下，均可实现金属/金属氧化物异质 结构. 其中, $\mathrm{ZnCoAl}-\mathrm{LDH}$ 为前体, 则在 $450^{\circ} \mathrm{C}$ 还原温 度下可得到 $\mathrm{Co} / \mathrm{Co}_{3} \mathrm{O}_{4}$ 纳米颗粒负载 $\mathrm{ZnO}-\mathrm{Al}_{2} \mathrm{O}_{3}$ 氧化物 
(a)

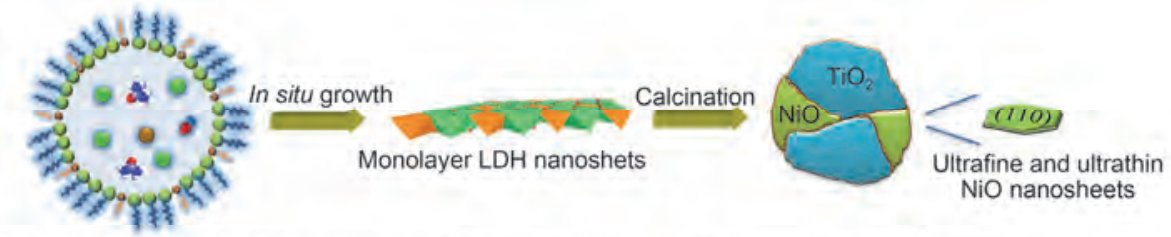

(b)
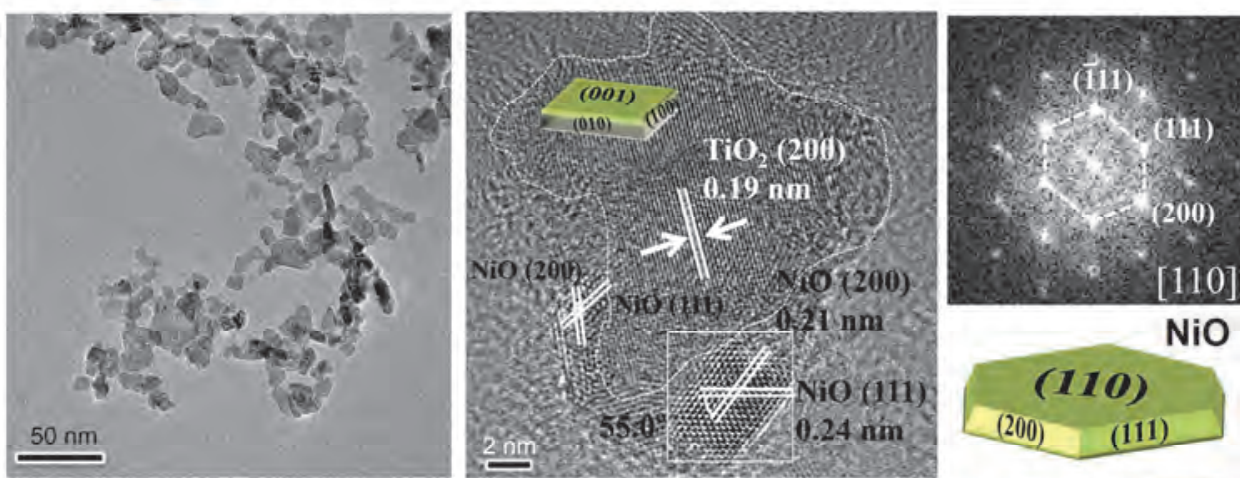

(c)
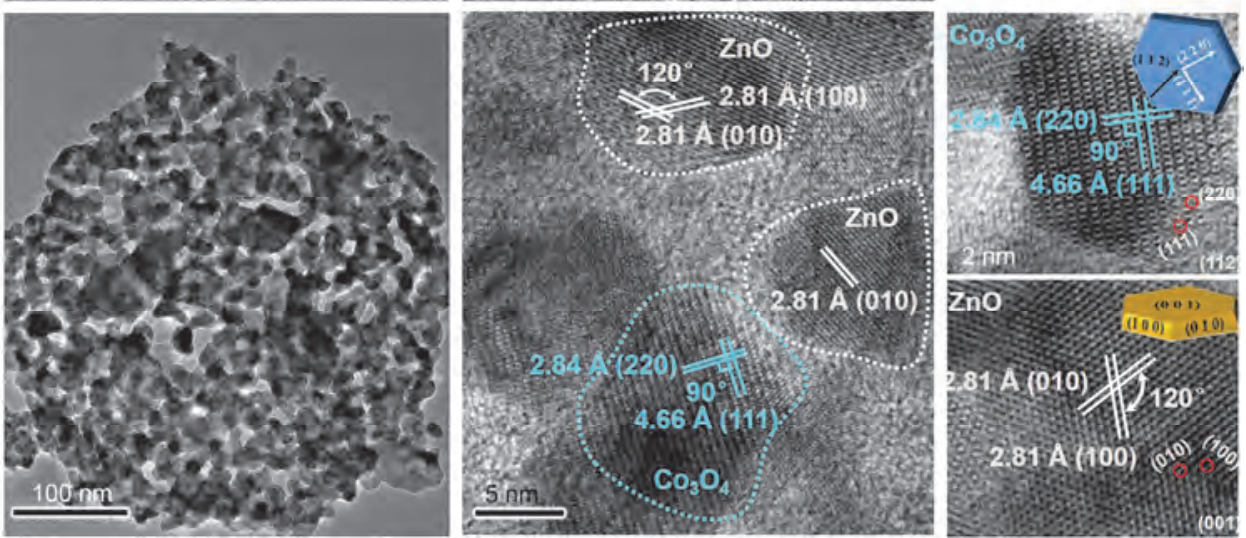

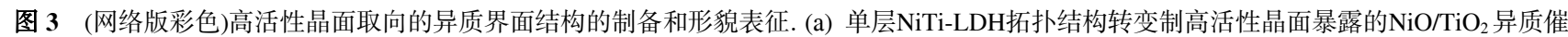
化剂的模型结构图 ${ }^{[22]}$; NiTi-MMO(b)及ZnCo-MMO(c) 的HRTEM图和FFT衍射斑点 ${ }^{[22,26]}$

Figure 3 (Color online) Preparation and morphologies characterization of heterogeneous interfacical structures with highly exposed facets. (a) Schematic illustration of the synthesis of ultrafine and ultrathin $\mathrm{NiO}$ nanosheets stabilized by $\mathrm{TiO}_{2}$ from monolayer NiTi-LDH nanosheet precursors ${ }^{[22]}$. HRTEM images and corresponding FFT patterns of NiTi-MMO (b) and ZnCo-MMO(c) ${ }^{[22,26]}$

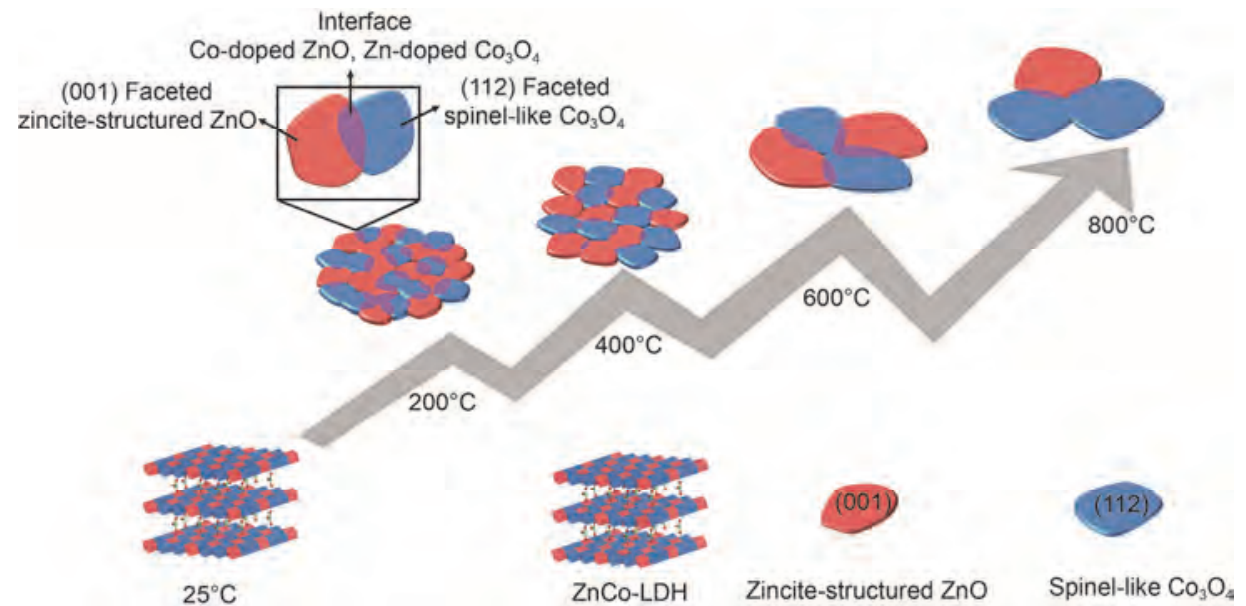

图 4 (网络版彩色) ZnCo-LDH拓扑转变调变异质结构界面过程 ${ }^{[27]}$

Figure 4 (Color online) Schematic illustration of the topological transformation from the ZnCo-LDH to the ZnCo- $x$ at different temperatures ${ }^{[27]}$ 

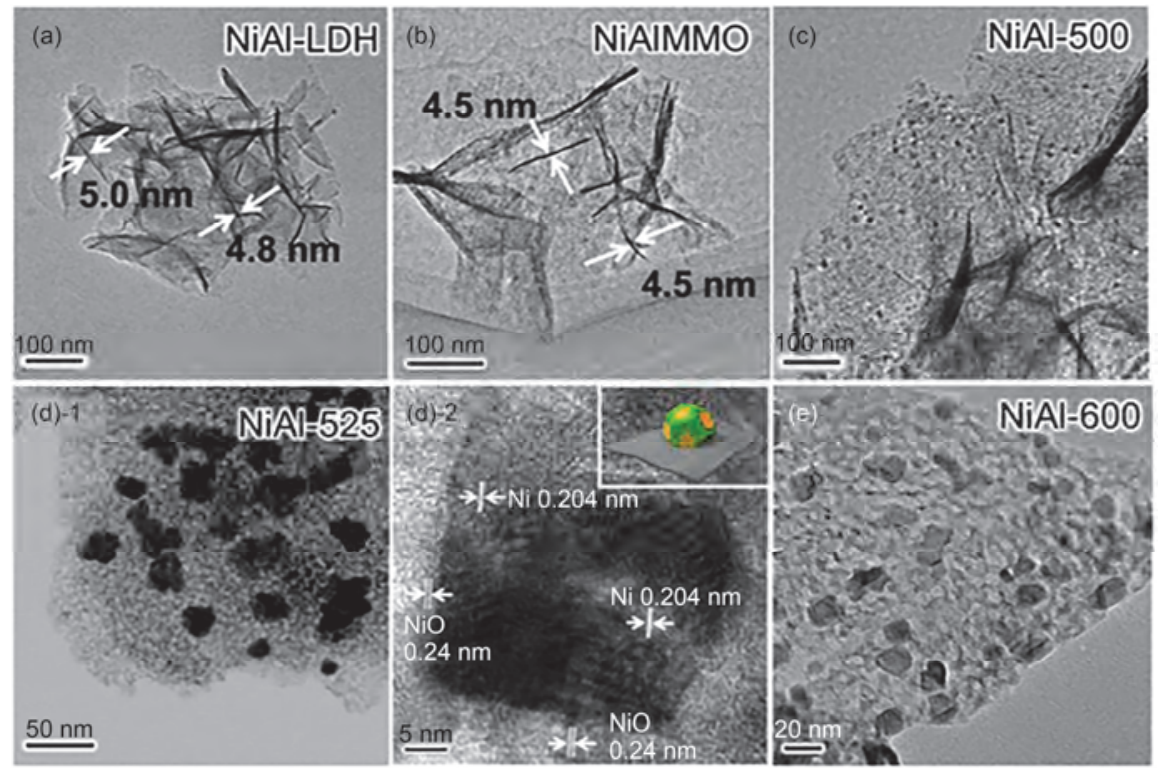

图 5 (网络版彩色)NiAl-LDH和NiAl-MMO的形貌表征. NiAl-LDH前体(a)和NiAl-MMO(b)的TEM图; (c) (e) NiAl-LDH在不同温度下经过还原 条件制备的 $\mathrm{Ni}$ 基催化剂的 TEM图 ${ }^{[29]}$

Figure 5 (Color online) Morphologies characterization of NiAl-LDH and NiAl-MMO. TEM images of NiAl-LDH precursors (a) and NiAl-MMO (b). (c)-(e) TEM images of NiAl-MMO reduced at different temperatures ${ }^{[29]}$

载体的催化剂. $\mathrm{Co}$ 和 $\mathrm{Co}_{3} \mathrm{O}_{4}$ 纳米颗粒间形成的异质结 弱化了 $\mathrm{Co}$ 催化剂的加氢能力, 提高了光驱动 $\mathrm{CO}$ 加氢 制备短链烯烃的选择性 ${ }^{[30]}$. 若以 $\mathrm{ZnFeAl}-\mathrm{LDH}$ 为前 体, 在 $500{ }^{\circ} \mathrm{C}$ 可控还原下可以得到 $\mathrm{Fe}^{0}$ 和 $\mathrm{FeO}_{x}$ 纳米颗 粒负载至 $\mathrm{ZnO}$ 和无定形 $\mathrm{Al}_{2} \mathrm{O}_{3}$ 上的异质结构光催化剂. 在该异质结构中 $\mathrm{Fe}^{0}$ 和 $\mathrm{FeO}_{x}$ 纳米颗粒之间存在着大量 的异质结界面, 为光催化费托反应提供优异的表面 电子结构, 从而可以有效调变反应产物的选择性 ${ }^{[31]}$.

\section{2 高分散 $\mathrm{CuCo}, \mathrm{CoFe}$ 合金催化剂的制备}

合金催化剂金属间的电子相互作用在催化反应 过程中会引起协同催化作用, 改变反应物及反应中 间物种的吸脱附性质, 进而提升催化剂的活性、选择 性及稳定性. 但是由于不同金属组分性质的差异, 如 何简易、绿色、可控地合成合金催化剂仍然具有挑战 性，而且金属间相互作用的复杂性限制了对其协同 催化作用的深人认识. LDHs材料独特的组成、结构特 性, 以及结构拓扑转变行为, 使其成为一种优异的合 金催化剂前体材料. Gao等人 ${ }^{[32]}$ 以 $\mathrm{CuCoAl}-\mathrm{LDH}$ 为前 体, 通过高温焙烧还原处理得到了 $\mathrm{Al}_{2} \mathrm{O}_{3}$ 负载的 $\mathrm{Cu} @ \mathrm{CuCo}$ 合金纳米颗粒. 该合金纳米颗粒在催化合 成气向高碳醇转化的反应中显示了优异的效果, 其 中 CO的转化率达到 $21.5 \%, \mathrm{C}_{6+}$ 的单元醇选择性可达
到 48.9\%. 张铁锐课题组 ${ }^{[33]}$ 利用共沉淀方法制备了 CoFeAl-LDH三元水滑石纳米片, 通过在氢气氛围内 可控还原处理，可以精确控制水滑石中金属的溢出 顺序, 得到了 3 种负载型催化剂, 其中还原温度低于 $400^{\circ} \mathrm{C}$ 得到的是 $\mathrm{FeO}_{x}$ 负载 $\mathrm{CoAl}-\mathrm{MMO}, 450 \sim 600^{\circ} \mathrm{C}$ 还原 得到 $\mathrm{FeO}_{x}-\mathrm{CoO}_{x}$ 负载无定型 $\mathrm{Al}_{2} \mathrm{O}_{3}$, 而 $650^{\circ} \mathrm{C}$ 以上即可 得到 $\mathrm{CoFe}$ 合金负载 $\mathrm{Al}_{2} \mathrm{O}_{3}$. 其还原顺序和金属还原难 易程度有关系. 这3 种负载型催化剂在光驱动 $\mathrm{CO}_{2}$ 加 氢过程中展现出不同的催化性能. 通过在LDHs前体 中引人不同金属, 系统调变LDHs前体结构拓扑转变, 可以有效实现不同合金结构，优化了合金的几何和 电子结构, 增强了双活性位的协同催化作用, 为高效 催化剂的结构设计与性能强化奠定了基础.

\section{3 含 $\mathrm{N}, \mathrm{Se}$ 等金属催化剂}

含有 $\mathrm{Ni}, \mathrm{Co}, \mathrm{Fe}$ 的LDHs或焙烧衍生的混合金属 氧化物在光、电催化反应中表现出良好的活性和稳定 性而被广泛报道, 但这些催化剂大多是半导体, 其导 电性仍不甚理想, 进一步阻碍了电子在催化剂中的 传导以及与电极之间的转移, 从而限制了其催化效 率. 相比上述的催化剂, 金属氮化物、锡化物等因其 独特的电子结构、优良的导电性和良好的耐腐蚀性 等, 有望提高其光、电解水的效率. 张铁锐课题组 ${ }^{[34]}$ 
通过微乳液法合成了超薄 $\mathrm{NiFe}-\mathrm{LDH}$ 纳米片前体, 高 温氨化得到了直径为 $100 \mathrm{~nm}$, 厚度为 $9 \mathrm{~nm}$ 的 $\mathrm{Ni}_{3} \mathrm{FeN}$ 纳米颗粒, 并对其电催化全分解水性能进行表征. $\mathrm{Ni}_{3} \mathrm{FeN}$ 纳米颗粒表现出优异的HER及OER催化活性, 催化剂的纳米尺寸效应增加了表面可利用的活性位 点, 也在一定程度上提高了催化效率. 进一步通过量 化计算, 从理论上解释了较其他单金属氮化物和氧 化物, 该化合物良好的导电性以及金属 $\mathrm{Fe}$ 元素外层 $\mathrm{d}$ 轨道的电子对提高 $\mathrm{H}_{2} \mathrm{O}$ 的吸附性的作用. 其优异的催 化性能取决于催化剂本身的金属特性与特殊的电子 结构, 在提高电导率的同时可以还增强了对反应物 分子 $\mathrm{H}_{2} \mathrm{O}$ 的吸附, 进而促进提高了其全分解水的性 能. 此外, 该策略可进一步拓展到 $\mathrm{Se}$ 化物体系, 利用 $\mathrm{NiCo}-\mathrm{LDH}$ 纳米阵列的转变和刻蚀处理的方法合成了 三维的 $\mathrm{Ni}_{1-x} \mathrm{Co}_{x} \mathrm{Se}_{2}$ 介孔网络结构纳米片 ${ }^{[35]}$, 该纳米 片在 $\mathrm{pH} 0 \sim 14$ 的范围内都显示了优异的电解水析氢活 性和结构稳定性. 上述工作采用LDHs为前体, 通过 不同氛围转变, 得到了高分散单金属、合金以及非金 属掺杂的纳米结构. 所制备的纳米结构除了具有高 分散、高负载密度等特点外, 其表面存在丰富的表界 面结构和结构缺陷, 为催化反应提供了丰富的活性 位点和高的电子传输能力.

\section{4 水滑石多级结构组装与传质扩散强化}

多级多孔材料因其富含微孔、介孔、大孔, 可以
有效促进反应客体分子的扩散，在催化、吸附和分离 方面有极其广泛的应用. 将多尺度孔结构组装为多 级结构, 可以在介观尺度进一步提升催化性能, 从而 可以实现催化性能的进一步优化. 凭借水滑石自身 独特的片层结构, Zhao等人 ${ }^{[36 ~ 38]}$ 选取自然界中易得 的仿生模板材料以及其他无机多级材料, 诸如豆荚、 滤纸、纤维、海绵、泡沫铝等作为载体, 采用原位生 长的方法，在上述载体表面原位获得了垂直取向的 $\mathrm{LDH}$ 多级结构 (图6). 即使在炦烧去除模板过程中, 其孔道结构也不会被破坏. 模板提供的多级孔道结 构以及LDH粒子的高分散特性，有利于反应物的扩 散与传质, 进而促进了其在光催化、吸附方面的性能.

\section{5 水滑石多尺度结构调控及光驱动催化 应用}

\section{1 光催化活化H-O键}

目前, 寻找合适的催化剂进行光/电分解水是材 料领域重要的研究方向, 其对于能源利用以及环境 保护方面具有重要意义 ${ }^{[39,40]}$. 自从 2009 年García课题 组 ${ }^{[41]}$ 首次将水滑石用于可见光条件下的光催化水解 产氧以来, 水滑石在光催化分解水方面的应用引起 了越来越多的关注 ${ }^{[42,43]}$. 2015 年, $\mathrm{YaO}$ 等人 ${ }^{[44]}$ 巧妙地 以 $\mathrm{ZnCr}-\mathrm{LDH}$ 为模板构筑了多级的花状 $\mathrm{CdZnS} @$ $\mathrm{ZnCr}-\mathrm{LDH}$ 复合材料, 并将其用于可见光解水产氢反
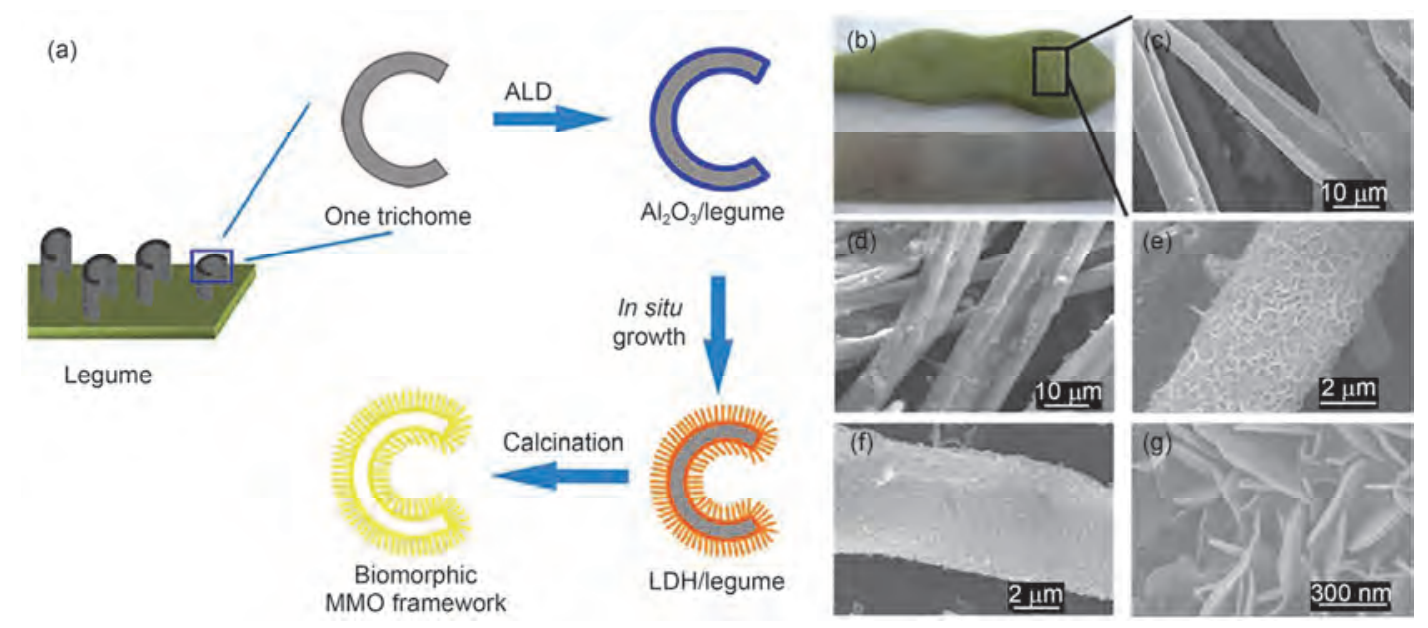

图 6 (网络版彩色)LDH及MMO薄膜的合成策略和形貌表征. (a) 用豆荚作生物模板合成LDH薄膜和MMO框架的示意图; (b) (g) 豆荚照片及 表面生长ZnAl-LDH和焙烧后得到的ZnAl-MMO的SEM图 ${ }^{[36]}$

Figure 6 (Color online) Synthesis strategy and morphologies characterization of LDH and MMO films. (a) Schematic representation for the fabrication of biotemplated LDH film and MMO framework from the legume. (b)-(g) Optical photograph of the legume; SEM images of the in situ growth $\mathrm{LDH} /$ legume film and ZnAl-MMO framework obtained by calcination of LDH/legume ${ }^{[36]}$ 
应中. 该多级结构所带来的催化剂活性位点的增加 以及电子空穴对的有效分离, 促进了光催化产氢效 率，其量子产率达到了 $4.13 \%$. 近年来，Zhao等人致 力于研究水滑石不同形貌在光/电催化水分解方面的 研究: 2012年 ${ }^{[14]}$, 采用共沉淀法合成了一系列的 $\mathrm{Ti}$ 基 水滑石, 发现MTi-LDH(M=Ni, Zn, Mg)具有优异的光 催化分解水产氢的性能. 由于水滑石层板上丰富的 $\mathrm{M}^{2+}-\mathrm{O}-\mathrm{Ti}$ 键, 使得 $\mathrm{TiO}_{6}$ 结构高度分散, 从而促进了 其光催化产氢性能; 2014年 ${ }^{[16]}$, 他们利用反相微乳法 合成了一系列不同晶粒尺寸的NiTi- LDH(30 90 nm), 发现 $\mathrm{Ti}^{3+}$ 缺陷的含量随着晶粒尺寸的减小而增大. 多 种表征结果显示这种丰富的表面 $\mathrm{Ti}^{3+}$ 缺陷可作为捕 获光生载流子的活性位点, 有效提高电子空穴的分 离效率, 从而在可见光下具有良好的光催化分解水 产氧性能 $(2148 \mu \mathrm{mol} /(\mathrm{g} \mathrm{h}))$, 在 $400 \mathrm{~nm}$ 处分别采用单 色照射, 量子产率可达65\%(图7). 在光催化过程中, 电催化剂往往作为光催化剂的助剂引起人们广泛关 注, 开发高效电催化剂, 可以有效提升光催化剂的性 能 ${ }^{[45]}$. 围绕上述单层 LDH纳米片, 进一步通过超声 剥离的方法, 张铁锐课题组 ${ }^{[18]}$ 合成了尺寸为 $1.5 \sim 3$ $\mathrm{nm}$, 厚度仅有 $0.6 \mathrm{~nm}$ 的超小超薄的镍铁水滑石 (NiFe-LDH-UF), 发现该材料的电催化产氧性能优于 普通 $\mathrm{NiFe}-\mathrm{LDH}$, 甚至超过了常用的 $\mathrm{IrO}_{2}$ 等贵金属氧 化物催化剂. 通过EXAFS表征以及DFT计算发现, 这 种NiFe-LDH-UF中具有丰富的金属和氧空位, 其结 构发生较大畸变, 具有半金属特性, 因此具有较好的 电催化产氧性能. 该研究结果为超小超薄水滑石的

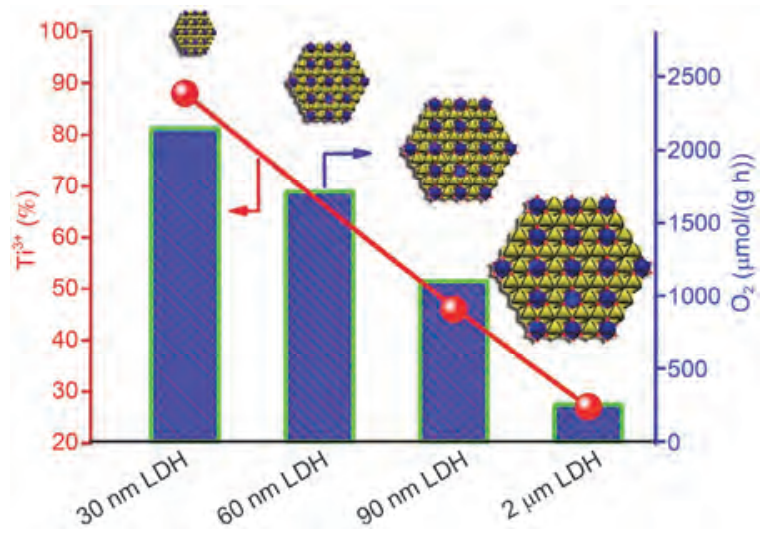

图 7 (网络版彩色) NiTi-LDH中 $\mathrm{Ti}^{3+}$ 的含量和光催化产氧效率随 LDH 粒径尺寸变化图 ${ }^{[16]}$

Figure 7 (Color online) The relationship between $\mathrm{Ti}^{3+}$ content and photocatalytic oxygen production efficiency with particle size of $\mathrm{Ni}$ Ti-LDH ${ }^{[16]}$
制备以及新型非贵金属电催化分解水材料提供了新 的思路 ${ }^{[21]}$. 将水滑石进一步煅烧拓扑结构转变为异质 氧化物结构 $\left(\mathrm{NiO} / \mathrm{TiO}_{2}, \mathrm{ZnO} / \mathrm{Co}_{3} \mathrm{O}_{4}\right)^{[22,27]}$, 其界面处提 供的丰富活性位点, 促进了电荷分离传输, 实现了低 电压下分解 $\mathrm{H}_{2} \mathrm{O}$ 产氧. 综上, 通过控制 $\mathrm{LDH}$ 的表面, 以及拓扑结构界面结构, 均可以实现高效活化 $\mathrm{H}_{2} \mathrm{O}$.

\section{2 光驱动催化活化 $\mathrm{C}=\mathrm{O}$ 键}

$\mathrm{CO}_{2}$ 的还原是吸热过程, 利用可再生能源提供反 应中所需能量, 是完成其转化的必要条件. 如何活化 稳定的 $\mathrm{CO}_{2}$ 分子, 是十分具有挑战性的课题. 与传统 的催化技术相比，在温和条件下光催化 $\mathrm{CO}_{2}$ 还原制备 碳氢化合物, 目前已成为新能源领域的重要研究方 向. LDHs纳米片具有的丰富可调变性使其成为一类 在 $\mathrm{CO}_{2}$ 转化中极具竞争力的光催化材料 ${ }^{[46 ~ 50]}$. 张铁 锐课题组 ${ }^{[21]}$ 利用简单的水热合成方法, 调控水滑石 纳米晶的生长微环境, 成功实现了超薄 $\mathrm{ZnAl}-\mathrm{LDH}$ 水 滑石纳米片的可控合成，其尺寸为 $40 \mathrm{~nm}$, 厚度约 2.7 $\mathrm{nm}$. 该超薄纳米片表面富含大量的氧缺陷位, 影响 了金属 Zn原子周围的电子轨道密度和配位环境, 形 成不饱和配位 $\mathrm{Zn}$. 该缺陷位可作为电子受限位, 有 利于光生电子传导到吸附在水滑石纳米片表面的反 应分子, 提高对 $\mathrm{CO}_{2}$ 吸附能力, 促进光催化 $\mathrm{CO}_{2}$ 还原 制备 $\mathrm{CO}$ 反应的进行. 但目前光催化 $\mathrm{CO}_{2}$ 还原的主要 产物为 $\mathrm{CO}, \mathrm{CH}_{4}$ 等, 如何有效地将 $\mathrm{CO}_{2}$ 进一步转化为 高热值的烃类, 成为亟待解决的问题. Co基催化剂具 有很高的催化活性、寿命长、加氢能力强等特点, 被 广泛应用于 $\mathrm{C} 1$ 化学转化制备高级碳氢化合物, 但其 缺点在于价格比较昂贵, 对温度比较敏感. Fe基催化 剂作为一种廉价的金属催化剂, 是目前加氢催化研 究的热点, 这类催化剂具有较宽的温度调变范围, 且 对产物的调控性较大. 结合 $\mathrm{Fe}$ 基催化剂较强的逆水 煤气变换反应活性 $\left(\mathrm{CO}_{2}+\mathrm{H}_{2}=\mathrm{CO}+\mathrm{H}_{2} \mathrm{O}\right)$ 和 $\mathrm{Co}$ 基催化剂 强的 $\mathrm{C}-\mathrm{C}$ 偶联能力, 张铁锐课题组 ${ }^{[33]}$ 以 CoFeAl-LDH 为前体, 经过简单的焙烧还原制得 $\mathrm{CoFe}$ 合金光催化 剂, 并将其应用于光催 $\mathrm{CO}_{2}$ 还原反应中. 通过不同温 度 $\left(300 \sim 700^{\circ} \mathrm{C}\right)$ 下焙烧还原处理, 可以调控催化剂的 结构组成, 进而影响产物的选择性(图 8(a)): 低温还 原得到的 $\mathrm{FeO}_{x}$ 基催化剂, $\mathrm{CO}_{2}$ 加氢还原产物为 $\mathrm{CO}$; 中 温还原得到的 $\mathrm{FeO}_{x}-\mathrm{CoO}_{x}$ 基催化剂, 产物主要为 $\mathrm{CH}_{4}$; 还原温度 $600{ }^{\circ} \mathrm{C}$ 得到的 $\mathrm{CoFe}$ 合金纳米颗粒负载于无 定型 $\mathrm{Al}_{2} \mathrm{O}_{3}$ 催化剂 $\left(\mathrm{CoFe}\right.$ alloy $\left./ \mathrm{Al}_{2} \mathrm{O}_{3}\right)$. 

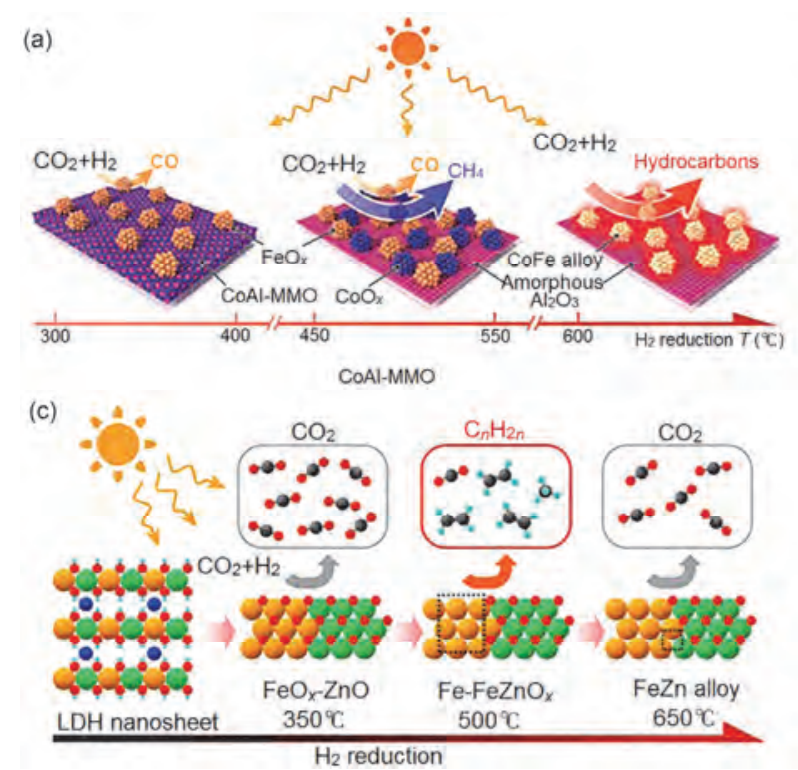

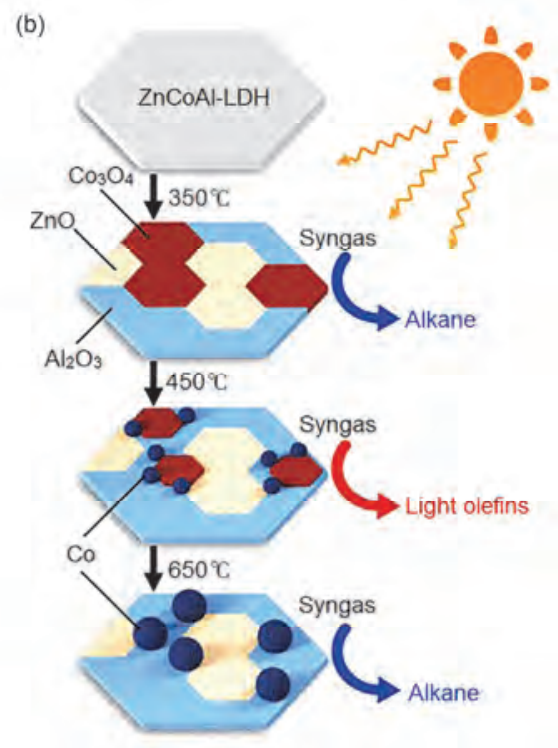

图 8 (网络版彩色) $\mathrm{CoFeAl}-\mathrm{LDH}(\mathrm{a})^{[33]}, \mathrm{ZnCoAl}-\mathrm{LDH}(\mathrm{b})^{[30]}$ 以及ZnFeAl-LDH(c) ${ }^{[31]}$ 不同温度下焙烧还原产物及其光驱动 $\mathrm{CO}_{2} / \mathrm{CO}$ 还原产物示意图 Figure 8 (Color online) Illustration of the different catalysts formed by hydrogen reduction of a CoFeAl-LDH (a) $)^{[33]}$, ZnCoAl-LDH (b) ${ }^{[30]}$ and $\mathrm{ZnFeAl}-\mathrm{LDH}(\mathrm{c})^{[3] 1}$ nanosheets precursor at different temperatures and their selectivity for $\mathrm{CO}_{2} / \mathrm{CO}$ hydrogenation under UV-vis excitation

其中, $\mathrm{CoFe}$ alloy $/ \mathrm{Al}_{2} \mathrm{O}_{3}$ 在紫外可见光光照 $2 \mathrm{~h}$ 后, $\mathrm{CO}_{2}$ 转化率可以高达 $78.6 \%$, 高碳烃化合物 $\mathrm{C}_{2+}$ 的选择 性首次达到 $35.2 \%$. 一系列对照实验和密度泛函理论 进一步证明 $\mathrm{CoFe}$ 合金更有利于 $\mathrm{C}-\mathrm{C}$ 键偶联, 进而实 现高附加值碳烃化合物的生成. 该工作首次报道了 光驱动 $\mathrm{CO}_{2}$ 加氢制高碳烃, 为利用 $\mathrm{CO}_{2}$ 原料生产高附 加值碳氢化合物提供了新的思路. 相比 $\mathrm{CO}_{2}, \mathrm{CO}$ 是重 要的煤制油原料. 通过对 $\mathrm{CO}$ 高温高压加氢可以实现 对高碳烃等化合物的生产. 该催化过程常用的催化 剂有 $\mathrm{Ni}, \mathrm{Co}, \mathrm{Fe}$ 等金属基催化剂. 如何实现温和条件 的CO催化加氢过程依旧是一个挑战. 2016年, Zhao等 人 ${ }^{[29]}$ 以 NiAl-LDH为前体, 通过高温还原制备了界面 丰富的 $\mathrm{NiO}_{x} / \mathrm{Ni}$ 异质结构, 在可见光下驱动 $\mathrm{CO}$ 加氢, 成功实现了 $\mathrm{C}-\mathrm{C}$ 偶联. 该异质结构中 $\mathrm{NiO}_{x}$ 层和 $\mathrm{Ni}$ 纳 米颗粒之间丰富的界面, 改变了 $\mathrm{NiO}_{x} / \mathrm{Ni}$ 纳米结构的 电子环境, 调变了 $\mathrm{CO}$ 氢化过程中间 $\mathrm{CH}_{2}$ * 物种的吸附 路径, 进一步促进了催化剂表面的 C-C偶联, 促进了 可见光催化费托反应制备高级烃类. 受该工作的启 发, Co基催化剂也具有类似的特性, 经水热法合成的 $\mathrm{ZnCoAl}-\mathrm{LDH}^{[30]}$, 可以高温可控还原为 $\mathrm{Co}_{3} \mathrm{O}_{4}$ 修饰的 $\mathrm{Co}$ 纳米颗粒, 该 $\mathrm{Co}$ 基异质催化体系在光热条件下, 实现了低碳烯烃的首次制备(图8(b)). 通过结构表征 和DFT理论计算发现氧修饰的Co颗粒调控了纳米结 构的电子环境, 该独特的结构更有利于 $\mathrm{CO}$ 的活化,
同时抑制了烯烃的进一步加氢作用. 近期张铁锐课 题组、马丁课题组和温晓东课题组 ${ }^{[31]}$ 合作, 采用双滴 法合成了 $\mathrm{ZnFeAl}-\mathrm{LDH}$, 并将其可控还原为 $\mathrm{FeO}_{x} / \mathrm{Fe}$ 纳 米催化剂, 研究发现表面的 $\mathrm{FeO}_{x}$ 层和 $\mathrm{Fe}$ 纳米颗粒间 丰富的界面, 改变了催化剂的电子微环境, $500^{\circ} \mathrm{C}$ 还 原的催化剂抑制 $\mathrm{CO}_{2}$ 生成, 且更有利于烯烃的生成(图 $8(\mathrm{c})$ ). 通过调控 $\mathrm{LDH}$ 不同金属种类以及还原温度, 可 控制备得到界面可调的异质催化剂. 在光驱动可控活 化 $\mathrm{C}=\mathrm{O}$ 方面, 利用 $\mathrm{LDH}$ 金属组成可调的特点, $\mathrm{CoFe}$ 合 金结构和金属/氧化物纳米异质结构 $\left(\mathrm{Ni} / \mathrm{NiO}_{x}\right.$, $\mathrm{Co} / \mathrm{Co}_{3} \mathrm{O}_{4}$ 和 $\mathrm{Fe} / \mathrm{FeO}_{x}$ ) 可有效调控 $\mathrm{C}-\mathrm{C}$ 键的耦合, 从而 实现高附加值碳烃化合物的催化合成.

\section{3 光催化活化 $\mathrm{N} \equiv \mathrm{N}$ 键}

氮元素是组成生物体的基本元素之一, 生物体 内的蛋白质、核酸等的合成都需要氮元素, 虽然 $\mathrm{N}_{2}$ 在空气中所占比例高达 $78 \%$, 但 $\mathrm{N}-\mathrm{N}$ 三键极其稳定, 不能被生物直接利用, 而植物生长所需的氮肥(如尿 素等)都需要以氨为原料. 目前, 工业合成氨仍采用 传统的 Haber-Bosch法, 该法主要利用 Fe 基催化剂, 在极其苛刻的反应条件下 $\left(15 \sim 25 \mathrm{MPa}, 300 \sim 500^{\circ} \mathrm{C}\right)$ 将 $\mathrm{N}_{2}$ 还原为 $\mathrm{NH}_{3}$, 该反应消耗大量的能源 (占全球能源 的 1\% 2\%), 而不可避免地造成 $\mathrm{CO}_{2}$ 的大量排放. 因 此, 开发一种绿色可持续的固氮方法十分必要, 光催 
化被认为是未来发展可再生能源的最佳途径之一 ${ }^{[51]}$. 在前期的研究中, 发现水滑石在光分解水、还原 $\mathrm{CO}_{2}$ 及 $\mathrm{C}-\mathrm{C}$ 偶联等方面有着优越的可调控性. 作为和 $\mathrm{CO}_{2}$ 一样稳定的分子, $\mathrm{N}_{2}$ 的活化和 $\mathrm{CO}_{2}$ 活化之间可能有着 类似的规律. 2017 年, 张铁锐课题组 ${ }^{[20]}$ 利用简单的共 沉淀法, 成功合成了一系列的超薄纳米催化剂 $M^{\mathrm{II}} \mathrm{M}^{\mathrm{II}}$ $-\operatorname{LDHs}\left(\mathrm{M}^{\mathrm{II}}=\mathrm{Mg}, \mathrm{Zn}, \mathrm{Ni}, \mathrm{Cu} ; \mathrm{M}^{\mathrm{II}}=\mathrm{Al}, \mathrm{Cr}\right)$, 并成功实现 了可见光催化 $\mathrm{N}_{2}$ 和水合成 $\mathrm{NH}_{3}$. 研究表明, 超薄 LDHs丰富的氧缺陷及扭曲的结构, 增强了对 $\mathrm{N}_{2}$ 的吸 附和光生电子从 $\mathrm{LDHs}$ 转移到 $\mathrm{N}_{2}$, 从而促进了 $\mathrm{NH}_{3}$ 的 合成, 特别是 $\mathrm{CuCr}-\mathrm{LDH}$ 在 $500 \mathrm{~nm}$ 的量子产率仍能达 到 $0.10 \%$, 该工作为常温常压下可见光催化合成 $\mathrm{NH}_{3}$ 提供了新路径.

\section{4 光催化处理有机污染物}

LDHs除了可以活化 $\mathrm{CO}_{2}, \mathrm{~N}_{2}$ 等这些十分稳定的 小分子外, 还可光催化降解有机污染物, 诸如罗丹 明、甲基蓝、甲基橙等 ${ }^{[52,53]}$. 2012年, Wei课题组 ${ }^{[54,55]}$ 通过电泳沉积法在铜箔上成功制备了定向生长的 $\mathrm{CuCr}-\mathrm{LDH}$ 薄膜, 该材料具有多孔结构、比表面积大、 在可见光区域(>400 $\mathrm{nm}$ ) 有较强吸收等特性. 通过改 变电泳电压, 可以精确地调节 $\mathrm{CuCr}-\mathrm{LDH}$ 薄膜的厚度. 厚度为 $16.5 \mu \mathrm{m}$ 的薄膜对降解 2,4,6-三氯苯酚、磺酰罗 丹明 $\mathrm{B}$ 和刚果红等污染物具有良好的光催化性能, 且 表现出优异的可循环性, 在多次回收使用后依然能 保持 $90 \%$ 以上的转化率. 基于LDHs的独特结构, 通 过在层板中引人不同金属离子, 调节LDHs的能带结 构, 可以有效调控其氧化/还原能力, 实现有机污染 物的部分氧化/还原, 乃至全氧化为 $\mathrm{CO}_{2}$, 为实现完全 降解有机污染物提供了一个催化剂平台.

综上所述, 利用LDHs 主体层板元素相互高分 散、晶粒尺寸和层板厚度可控、自身在结构转变过程 中具有拓扑转变的特性等结构特点, 构筑了一系列 LDHs基及 LDHs为前体的催化材料. 通过调变 LDHs 纳米片主体层板元素种类及比例, 调节其能带和缺 陷位, 进而实现水滑石及其其焙烧产物对光谱吸收 能力以及价导带位置调整的目的, 同时其丰富的缺
陷位会提供大量的催化活性位点, 有助于提高光生 载流子利用率. 利用 $\mathrm{LDHs}$ 纳米片层板堆叠数和粒径 大小可控的特点, 可以合成不同大小和厚度的水滑 石纳米片, 可以精准控制氧缺陷的浓度. 以组成以及 结构形貌可控的LDHs纳米片为光催化剂前体, 经过 高温炦烧过程后可实现对界面结构的调控进而优化 活性位点数量, 达到高效活化反应物的目的. 通过 LDHs多尺度结构的精准调控, 目前已经实现了 $\mathrm{H}_{2} \mathrm{O}$ 分 子中 $\mathrm{O}-\mathrm{H}$ 键、 $\mathrm{CO}_{2}$ 分子中 $\mathrm{C}=\mathrm{O}$ 键以及 $\mathrm{N}_{2}$ 分子中 $\mathrm{N} \equiv \mathrm{N}$ 的 选择性催化活化和有机污染物的高效降解 $(\text { 表 } \mathrm{S} 1)^{[56]}$.

\section{6 结论和展望}

研究人员通过调控LDHs的晶粒尺寸、层板厚度、 合成方法等，对LDHs微观精细结构、介观异质界面 结构、高分散催化剂分散特性、不同缺陷种类浓度以 及晶体结构扭曲畸变等调变, 从而达到对LDHs缺陷 结构、能带结构以及光生载流子分离传输效率的优化 调控. 这些LDHs往往具有较大的比表面积、较多的 活性位点、较为丰富的缺陷结构以及经过拓扑转变制 备异质结构, 这种丰富多样的可调变特性, 使得 $\mathrm{LDHs}$ 基纳米材料在光驱动还原 $\mathrm{CO}_{2}, \mathrm{CO}$ 加氢制高碳 烃、合成氨以及有机污染物处理等方面展示了强大 的生命力, 从而为工业上实现光催化高效转化提供 思路.

然而，目前LDHs基光催化材料还存在如下问题: 在合成方面, 更小尺寸(<1.5 nm) 以及更大尺寸(可供 测试单晶衍射)的水滑石还有待突破; 缺乏先进的表 征技术手段来准确表征结构调变过程中活性位的产 生和演变规律 (例如不同的缺陷和异质结界面的变 化), 如原位监测催化剂和反应物的相互作用方式及 构效关系, 揭示可能的催化反应机理并用于指导催 化剂的设计和制备等. 在催化应用方面, 研究者仍面 临着诸如: 如何利用 $L D H s$ 将 $\mathrm{CO}_{2}$ 还原直接得到高碳 烃; 如何提高 $\mathrm{LDHs}$ 光催化合成氨的量子产率等挑战. 相信在未来, 以水滑石为主体的应用领域会逐步拓 宽, 不同晶粒尺寸、层板厚度、缺陷类型、电子结构 的LDHs会受到更加广泛的关注和研究.

\section{参考文献}

1 Yang N, Yu A B, Li J H. Preface to multiscale structures and systems in process engineering special issue. Ind Eng Chem Res, 2013, 52: $11225-11227$ 
2 Li J H. Exploring the logic and landscape of the knowledge system: Multilevel structures, each multiscaled with complexity at the mesoscale. Engineering, 2016, 2: 276-285

3 Sideris P J, Nielsen U G, Gan Z, et al. Mg/Al ordering in layered double hydroxides revealed by multinuclear NMR spectroscopy. Science, 2008, 321: 113-117

4 Nishimura S, Takagaki A, Ebitani K. Characterization, synthesis and catalysis of hydrotalcite-related materials for highly efficient materials transformations. Green Chem, 2013, 15: 2026-2042

5 Fan G L, Li F, Evans D G, et al. Catalytic applications of layered double hydroxides: Recent advances and perspectives. Chem Soc Rev, 2014, 43: 7040-7066

6 Feng J T, He Y F, Liu Y N, et al. Supported catalysts based on layered double hydroxides for catalytic oxidation and hydrogenation: General functionality and promising application prospects. Chem Soc Rev, 2015, 44: 5291-5319

7 Markov L, Petrov K. Topotactic preparation of copper-cobalt oxide spinels by thermal decomposition of double-layered oxide hydroxide nitrade mixed crystals. Solid State Ionics, 1990, 39: 187-193

8 Xiang X, Xie L S, Li Z W, et al. Ternary $\mathrm{MgO} / \mathrm{ZnO} / \mathrm{In}_{2} \mathrm{O}_{3}$ heterostructured photocatalysts derived from a layered precursor and visible-light-induced photocatalytic activity. Chem Eng J, 2013, 221: 222-229

9 Ma R Z, Liu Z P, Li L, et al. Exfoliating layered double hydroxides in formamide: A method to obtain positively charged nanosheets. J Mater Chem B, 2006, 16: 3809-3813

10 Yu J F, Wang Q, O'Hare D, et al. Preparation of two dimensional layered double hydroxide nanosheets and their applications. Chem Soc Rev, 2017, 46: 5950-5974

11 Zhao Y F, Jia X D, Waterhouse G I N, et al. Layered double hydroxide nanostructured photocatalysts for renewable energy production. Adv Energy Mater, 2016, 6: 1501974-1501993

12 Yin H J, Tang Z Y. Ultrathin two-dimensional layered metal hydroxides: An emerging platform for advanced catalysis, energy conversion and storage. Chem Soc Rev, 2016, 45: 4873-4891

13 Wang Q, O'Hare D. Recent advances in the synthesis and application of layered double hydroxide (LDH) nanosheets. Chem Rev, 2012, 112: 4124-4155

14 Zhao Y F, Chen P Y, Zhang B S, et al. Highly dispersed $\mathrm{TiO}_{6}$ units in a layered double hydroxide for water splitting. Chem Eur J, 2012, 18: 11949-11958

15 Zhao Y F, Wang C J, Gao W, et al. Synthesis and antimicrobial activity of ZnTi-layered double hydroxide nanosheets. J Mater Chem B, 2013, 1: 5988-5994

16 Zhao Y F, Li B, Wang Q, et al. NiTi-layered double hydroxides nanosheets as efficient photocatalysts for oxygen evolution from water using visible light. Chem Sci, 2014, 5: 951-958

17 Zhao Y F, Wang Q, Bian T, et al. $\mathrm{Ni}^{3+}$ doped monolayer layered double hydroxide nanosheets as efficient electrodes for supercapacitors. Nanoscale, 2015, 7: 7168-7173

18 Zhao Y F, Zhang X, Jia X D, et al. Sub-3 nm ultrafine monolayer layered double hydroxide nanosheets for electrochemical water oxidation. Adv Energy Mater, 2018, 8: 1703585-17035912

19 Sun Z H, Liu Q H, Yao T, et al. X-ray absorption fine structure spectroscopy in nanomaterials. Sci China Mater, 2015, 58: 313-341

20 Zhao Y F, Zhao Y X, Waterhouse G I N, et al. Layered-double-hydroxide nanosheets as efficient visible-light-driven photocatalysts for dinitrogen fixation. Adv Mater, 2017, 29: 1703828-1703837

21 Zhao Y F, Chen G B, Bian T, et al. Defect-rich ultrathin $\mathrm{ZnAl}^{-1 a y e r e d ~ d o u b l e ~ h y d r o x i d e ~ n a n o s h e e t s ~ f o r ~ e f f i c i e n t ~ p h o t o r e d u c t i o n ~ o f ~} \mathrm{CO}_{2}$ to CO with water. Adv Mater, 2015, 27: 7824-7831

22 Zhao Y F, Jia X D, Chen G B, et al. Ultrafine NiO nanosheets stabilized by $\mathrm{TiO}_{2}$ from monolayer NiTi-LDH precursors: An active water oxidation electrocatalyst. J Am Chem Soc, 2016, 138: 6517-6524

23 Zhao X F, Zhang Y C, Xu S L, et al. Oriented $\mathrm{CoFe}_{2} \mathrm{O}_{4} / \mathrm{CoO}$ nanocomposite films from layered double hydroxide precursor films by calcination: Ferromagnetic nanoparticles embedded in an antiferromagnetic matrix for beating the superparamagnetic limit. J Phys Chem C, 2012, 116: 5288-5294

24 Zhao X F, Zhang F Z, Xu S L, et al. From layered double hydroxides to ZnO-based mixed metal oxides by thermal decomposition: Transformation mechanism and UV-blocking properties of the product. Chem Mater, 2010, 22: 3933-3942

25 He S, Zhang S T, Lu J, et al. Enhancement of visible light photocatalysis by grafting ZnO nanoplatelets with exposed (0001) facets onto a hierarchical substrate. Chem Commun, 2011, 47: 10797-10799

$26 \mathrm{Xu} \mathrm{Y} \mathrm{Q}$, Wang Z L, Tan L, et al. Interface engineering of high-energy faceted $\mathrm{Co}_{3} \mathrm{O}_{4} / \mathrm{ZnO}$ heterostructured catalysts derived from layered double hydroxide nanosheets. Ind Eng Chem Res, 2018, 57: 5259-5267

27 Xu Y Q, Wang Z L, Tan L, et al. Fine tuning the heterostructured interfaces by topological transformation of layered double hydroxide 
nanosheets. Ind Eng Chem Res, 2018, 57: 10411-10420 CdZnS@LDH microstructures with improved visible-light-driven $\mathrm{H}_{2}$ production. Chem Asian J, 2015, 10: 630-636 photoelectrochemical water splitting. Adv Funct Mater, 2014, 24: 580-586

47 Teramura K, Iguchi S, Mizuno Y, et al. Photocatalytic conversion of $\mathrm{CO}_{2}$ in water over layered double hydroxides. Angew Chem Int Ed, 2012, 51: 8008-8011

48 Iguchi S, Hasegawa Y, Teramura K, et al. Preparation of transition metal-containing layered double hydroxides and application to the photocatalytic conversion of $\mathrm{CO}_{2}$ in water. $\mathrm{J} \mathrm{CO}_{2}$ Util, 2016, 15: 6-14

49 Tonda S, Kumar S, Bhardwaj M, et al. g- $\mathrm{C}_{3} \mathrm{~N}_{4} / \mathrm{NiAl}-\mathrm{LDH}$ 2D/2D hybrid heterojunction for high-performance photocatalytic reduction of $\mathrm{CO}_{2}$ into renewable fuels. ACS Appl Mater Interfaces, 2018, 10: 2667-2678

50 Lv M, Liu H. Photocatalytic property and structural stability of CuAl-based layered double hydroxides. J Solid State Chem, 2015, 227: 232-238

51 Li H, Shang J, Ai Z H, et al. Efficient visible light nitrogen fixation with BiOBr nanosheets of oxygen vacancies on the exposed \{001\} facets. J Am Chem Soc, 2015, 137: 6393-6399

52 Liu X, Zhao X F, Zhu Y, et al. Experimental and theoretical investigation into the elimination of organic pollutants from solution by layered double hydroxides. Appl Catal B Environ, 2013, 140-141: 241-248 
53 Shao M F, Han J B, Wei M, et al. The synthesis of hierarchical Zn-Ti layered double hydroxide for efficient visible-light photocatalysis. Chem Eng J, 2011, 168: 519-524

54 Tian L, Zhao Y F, He S, et al. Immobilized Cu-Cr layered double hydroxide films with visible-light responsive photocatalysis for organic pollutants. Chem Eng J, 2012, 184: 261-267

55 Zhao Y F, Zhang S T, Li B, et al. A family of visible-light responsive photocatalysts obtained by dispersing $\mathrm{CrO}_{6}$ octahedra into a hydrotalcite matrix. Chem Eur J, 2011, 17: 13175-13181

56 Shao M F, Wei M, Evans D G, et al. Layered double hydroxide materials in photocatalysis. In: Yan D, Wei M, eds. Photofunctional Layered Materials. Cham: Springer, 2015. 166: 105-136

\section{补充材料}

图 S1 CuCr-LDH 的结构示意图和结构表征

图 S2 ZnAl-LDH 的结构示意图和表征

图 S3 NiAl-MMO 和 Ni- $x$ 的原位 XRD, UV/Vis 光谱, XANES 和 EXAFS 谱以及原位 XPS 图谱

表 S1 LDHs 及 LDHs 为前体的太阳能驱动催化材料在多种催化反应中的应用

本文以上补充材料见网络版 csb.scichina.com. 补充材料为作者提供的原始数据, 作者对其学术质量和内容负责. 


\title{
Multiscale structural modulation of layered double hydroxide nanosheets and their application in solar-driven catalysis
}

\author{
Yanqi Xu ${ }^{1}$, Ling $\operatorname{Tan}^{1}$, Zelin Wang ${ }^{1}$, Xiaojie Hao ${ }^{1}$, Jikang Wang ${ }^{1}$, Yufei Zhao ${ }^{1 *} \&$ Yu-Fei Song ${ }^{1,2 *}$ \\ ${ }^{1}$ State Key Laboratory of Chemical Resource Engineering, Beijing University of Chemical Technology, Beijing 100029, China; \\ ${ }^{2}$ Beijing Advanced Innovation Center for Soft Matter Science and Engineering, Beijing 100029, China \\ * Corresponding authors, E-mail: zhaoyufei@mail.buct.edu.cn; songyf@ mail.buct.edu.cn
}

Due to the high photocatalytic efficiency in activating chemical bonding under mild reaction conditions, photocatalysis has been acted as a green and promising technology. However, how to improve the quantum efficiency and solar energy utilization to further enhance the photocatalytic performance remains a challenge, and it is highly desirable to rational synthesize photocatalyst with suitable energy band structure, and efficient active sites.

Layered double hydroxides (LDHs) is a kind of two-dimensional materials, with molecular formula $\left[\mathrm{M}_{1-x}^{2+} \mathrm{M}_{x}^{3+}(\mathrm{OH})_{2}\right]^{x+}$ $\left[\mathrm{A}_{x / n}\right]^{n-} \cdot \mathrm{mH}_{2} \mathrm{O}$. The layered structure of LDHs is similar to brucite $\mathrm{Mg}(\mathrm{OH})_{2}$, which is connected by the octahedral $\mathrm{MO}_{6}$ sites. The substitution of divalent metal cations $\left(\mathrm{M}^{2+}\right)$ with trivalent metal cations $\left(\mathrm{M}^{3+}\right)$ makes the layers positively charged, in which $\mathrm{M}^{2+}, \mathrm{M}^{3+}$ can be as one or more of the elements such as $\mathrm{Mg}, \mathrm{Al}, \mathrm{Ti}, \mathrm{V}, \mathrm{Cr}, \mathrm{Mn}, \mathrm{Fe}, \mathrm{Co}, \mathrm{Ni}, \mathrm{Cu}, \mathrm{Zn}, \mathrm{Ga}$, In, etc. $\mathrm{M}^{2+}, \mathrm{M}^{3+}$ ions alternately arrange in the main layers at a high atomic level to form the planar structure $(a, b$ axis direction), and the alternation of the positively charged layers and the negatively charged anionic layers ( $c$ axis direction) constitute the three-dimensional structure of LDHs. There are many unique structural characteristic of LDHs, such as the high dispersion of the metal elements in the layers, the controllable particle size and thickness, topological transformation and so on. The above properties have provide LDH as photocatalysts as the followings reason. Firstly, the metal elements of LDHs are arranged in an alternating order at the atomic level with flexible compositions (such as $\mathrm{Fe}, \mathrm{Co}, \mathrm{Ni}, \mathrm{Ti}$, etc.), and can be directly used as photocatalysts due to the tunable band gap. Secondly, the topological transformation property can turn LDH materials into heterogeneous mixed metal oxides or highly supported metal-containing catalysts, which are not easily agglomerated even under reaction condition. Thirdly, based on the tunable particle size and thickness of LDHs, it is possible to adjust the morphology of LDHs into nanoscale, which can fully expose the active sites and enhance the catalytic performance. Based on the abundant adjustable properties of LDHs, the following structures can be well optimized, such as their composition, particle sizes, defects, abundant interfaces, the high dispersion characteristics, and tunable energy band structures, etc. by using LDHs as catalysts for the potential enhanced catalytic efficiency.

In this review, in order to activate $\mathrm{H}-\mathrm{O}, \mathrm{C}=\mathrm{O}, \mathrm{N} \equiv \mathrm{N}$ and $\mathrm{C}-\mathrm{C}$ bonds under mild condition, a series of LDH-based photocatalysts have been finely synthesized (like NiTi-LDH nanosheets, ZnAl-LDH, CoFe alloy derived from LDH, $\mathrm{Co} / \mathrm{Fe}$-hetrostructure derived from $\mathrm{LDH}, \mathrm{Cr}$-containing $\mathrm{LDH}$, etc.), and widely applied in solar-driven water splitting, $\mathrm{CO}_{2}$ reduction, $\mathrm{CO}$ hydrogenation to high carbon hydrocarbons, ammonia synthesis, degradation and so on. Collectively, with the multiscale structural regulation of the microstructure, mesoscopic interfacial structure and highly dispersed metal-supported structure, the following properties such as the band gap, defect and the interface of LDH-based photocatalysts can be well modulated, and the solar-driven catalytic performance are demonstrated to be comprehensively facilitated. Furthermore, the relationship among the preparation, structure and catalysis has been well discussed, which will provide a strategy for the rational design of efficient photocatalysts.

layered double hydroxides, multiscale structural regulation, defect, interface, photocatalysis, photothermal catalysis

doi: 10.1360/N972018-00839 